\title{
Blending of Designer Synthetic Polymers to a Dual Targeted Nanoformulation for SARS-CoV-2 Associated Kidney Damage
}

\author{
Bapurao Surnar, Anuj S. Shah, Subham Guin, Nagesh Kolishetti, Alessia Fornoni, and Shanta Dhar* \\ Cite This: https://doi.org/10.1021/acs.biomac.1c00799 \\ Read Online
}

ABSTRACT: As the COVID-19 pandemic has continued to spread, studies have shown that hospitalized COVID-19 patients are at significant risk for developing acute kidney injury (AKI), which can cause increased morbidity, the need for dialysis treatment, chronic kidney diseases, and even death. In this paper, we present a proof-of-concept study for the utilization of combination therapeutic-loaded dual-targeted biodegradable nanoparticles (NPs) to treat concurrent AKI and COVID-19 in patients by delivering the therapeutics across the gut epithelial barrier and to the kidney, in order to lower the viral load as well as reduce the symptoms of AKI. Despite recent vaccination efforts and the end of the COVID-19 pandemic in sight, problems related to the longterm effects of COVID-19 will continue to persist, including impacts on patients suffering from $\mathrm{AKI}$ and other chronic renal conditions. Therefore, the dual-targeted blended polymeric NP developed in this study to treat concurrent COVID-19 infection and AKI is a useful proof-ofconcept nanoplatform for future treatments of these complications.

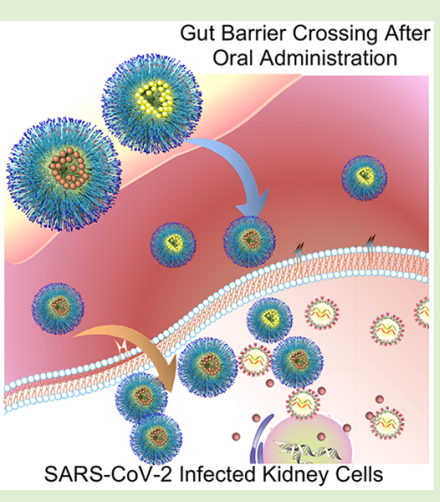

\section{INTRODUCTION}

After over a year of the COVID-19 pandemic, with particularly significant infection and death rates across the Globe, COVID19 and its associated comorbidities have killed over three million people. Although over 1 billion doses of COVID-19 vaccines have been given out globally, devastating recent outbreaks have emerged in countries such as India and Brazil, and uncertainty is still present regarding the length of immunity conferred by the vaccines and the potential need for additional booster doses in the fall months. Using its spike proteins to bind to the angiotensin converting enzyme 2 (ACE2) receptor, the severe acute respiratory syndrome coronavirus 2 (SARS-CoV-2) infects respiratory epithelial cells which are involved in blood pressure regulation and can inactivate cellular alarm pathways such as interferon production. $^{1,2}$ In addition to the enormous public health burden, the global pandemic has generated enormous anxiety, which has impacted every sector of life and industry and resulted in a significant toll on the economy. This has been worsened by the growing list of comorbidities associated with COVID-19, which arise through the failure of crucial organs such as the lungs, heart, brain, and kidney. COVID-19 infection can cause multiple side effects that contribute to the onset of acute kidney injury (AKI). A recent study shows over $24 \%$ of the hospitalized COVID-19 patients have AKI and this number increases to $61 \%$ for ICU patients. ${ }^{3}$ Kidney and glomerular cells contain ACE2 receptors and are susceptible to SARS-CoV-2 virus entry and infection. ${ }^{4}$ Although some strides have been made in characterizing the causal relationship between COVID-19 infection and AKI, the causative mechanism of this pathology is not understood, and there is currently no effective treatment. However, recent studies have also shown that the presence of AKI in patients also renders them more susceptible to more severe COVID-19 infection and increased fatality due to the coronavirus and development of chronic kidney diseases.

AKI involves the sudden onset of kidney damage or failure within a short period of time and can be characterized by decreased blood flow to the kidneys, kidney tubular damage and inflammation, vasculitis, and the possibility of sepsis and death, all effects that can be caused or worsened by COVID$19 .^{6-10}$ The presence of AKI consistently worsens outcomes for patients with a variety of other conditions, including myocardial infarction and heart disease, cirrhosis, and viral diseases like COVID-19. ${ }^{11-14}$ Furthermore, among patients with concurrent AKI and COVID-19 infection, compared to early stage AKI, late-stage AKI was more closely associated with higher levels of inflammatory markers and increased mortality. 15

In this paper, we present well-integrated chemistry and engineering approaches to provide an orally administrable, biocompatible, dual-targeted, polymeric, blended nanoparticle (NP) to deliver an FDA-approved antiviral drug, ivermectin (IVM), and a well-known antioxidant dietary supplement, coenzyme Q10 (CoQ $\left.{ }_{10}\right)$, for COVID-19 treatment and

Received: June 23, 2021

Revised: $\quad$ August 9, 2021 
associated problems related to AKI (Figure 1). The Fc targeting component of this engineered NP system allows for

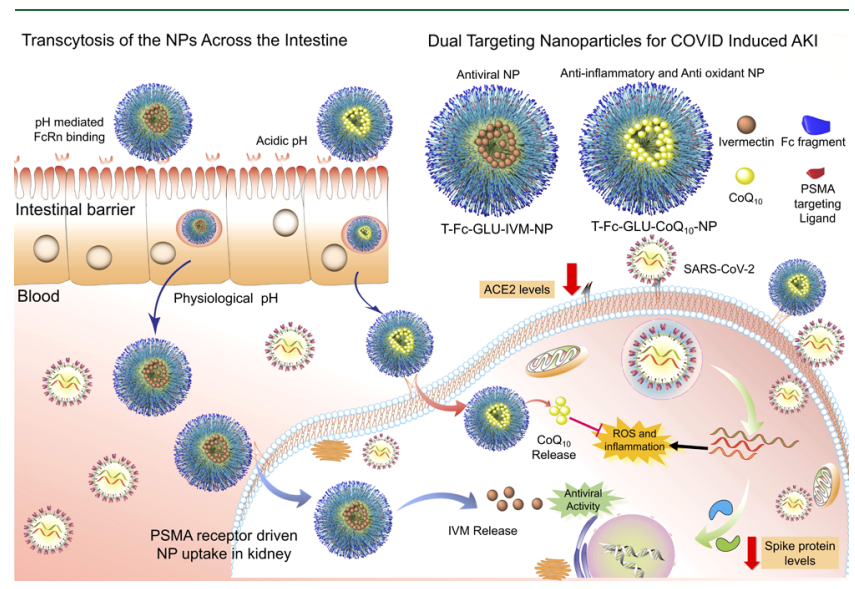

Figure 1. Schematic representation of dual-targeting IVM- and $\mathrm{CoQ}_{10}$-loaded NPs. The FcRn-mediated transcytosis through the gut epithelial barrier followed by circulation and localization at kidney via PSMA-mediated uptake into kidney cells, and release of IVM and $\mathrm{CoQ}_{10}$ to lower expression of ACE2 and spike protein as well as decrease inflammation and ROS levels in the kidney cells.

transportation of the NP through the gut endothelial barrier, the entry pathway of the virus, and delivery of the antiviral drug to the gut as well as in circulation to tackle the virus infected cells. The other targeting component drives the antiviral and the antioxidant to the kidney to reduce the virus infected cells and reverse the effects of the virus by reducing oxidative stress and inflammation (Figure 1). This platform was developed by utilizing the understanding of different drug and polymer solubilities and molecular weight of the polymers, and utilizing the receptors which are expressed at the gut and kidney endothelia and proximal tubules of the kidney.

\section{EXPERIMENTAL SECTION}

Materials and Methods. Description of materials and all methods are included in the Supporting Information.

Animals. Balb/c Albino mice were purchased from Jackson Laboratory. All animals were handled in accordance with "The Guide for the Care and Use of Laboratory Animals" of American Association for Accreditation of Laboratory Animal Care (AAALAC), Animal Welfare Act (AWA), and other applicable federal and state guidelines. All animal work presented here was approved by Institutional Animal Care and Use Committee (IACUC) of University of Miami Miller School of Medicine. Animals had free access to chow diet and water during all experiments.

\section{RESULTS AND DISCUSSION}

Gut Epithelial Barrier- and Kidney-targeting Blended Nanoparticle. Recently, we have developed an orally administrable, biodegradable IVM-loaded NP which has the potential to be used for antiviral therapy. ${ }^{16,17}$ These NPs, composed of poly(lactide-co-glycolide)- $b$-poly(ethylene glycol)-maleimide (PLGA- $b-\mathrm{PEG}_{2000}-\mathrm{Mal}$ ) polymer for conjugation with $\mathrm{Fc}$ fragment to target the neonatal $\mathrm{Fc}$ receptor $(\mathrm{FcRn})$ for transport of NPs to the bloodstream after oral delivery, showed improved delivery of IVM into cells. ${ }^{16,17}$ The FcRn mediates immunoglobulin $\mathrm{G}$ ( IgG) transport across the polarized epithelial barriers utilizing a $\mathrm{pH}$-driven pathway. ${ }^{18-20}$ For the current purpose, we synthesized the polymer PLGA- $b$ $\mathrm{PEG}_{6000}-\mathrm{Mal}$ with $\mathrm{PEG}_{6000}$ (Figure 2). The polymers were characterized using NMR and GPC (Figure S1-S5). Prostate specific membrane antigen (PSMA) is expressed on proximal tubules of the kidney, and its expression is at increased levels on the kidney following kidney diseases, renal cancer, or other tissue damage due to the presence of tissue regrowth and neoplasms. ${ }^{21-25}$ Thus, we developed a biodegradable polymer,
(A)

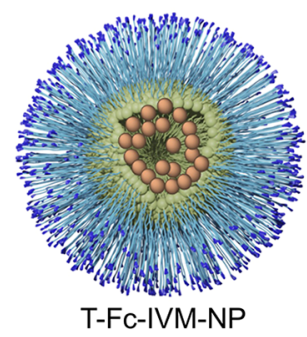

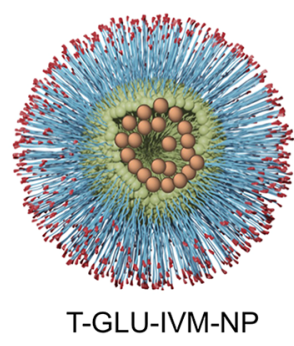

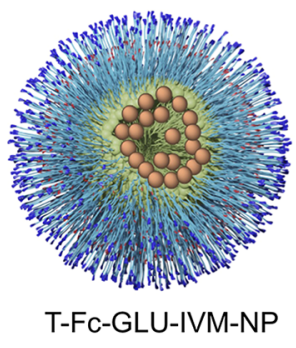

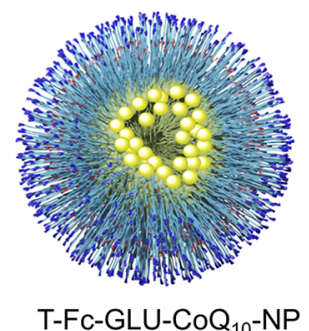

(C)
(B)<smiles>NCCCCCC(=O)OCCCCn1c(=O)ccc(=O)n1CCCCC(=O)ON1C(=O)CCC1=O</smiles>
$84 \%$

$$
\text { (a) }
$$

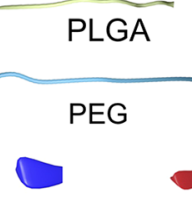

Fc fragment GLU

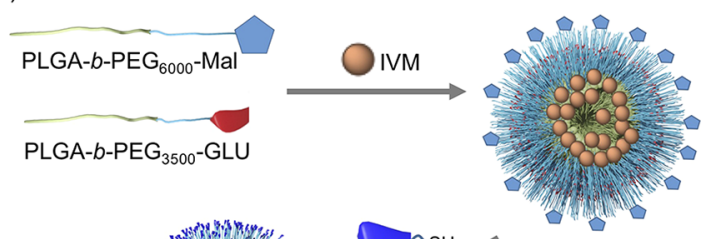

Figure 2. (A) Different blended NPs which were used in this study. (B) Synthesis of PLGA- $b$-PEG $6000-\mathrm{Mal}_{\text {from }}$ Mal-NHS and PLGA- $b$-PEG $6000^{-}$ $\mathrm{NH}_{2}$. (C) Synthesis of IVM-loaded blended NPs made from PLGA- $b$-PEG $3500-$ GLU and PLGA- $b$-PEG $6000-$ Mal, followed by conjugation of the Fc immunoglobulin fragment via sulfhydryl immobilization, producing dual-targeted T-Fc-GLU-IVM-NPs. 


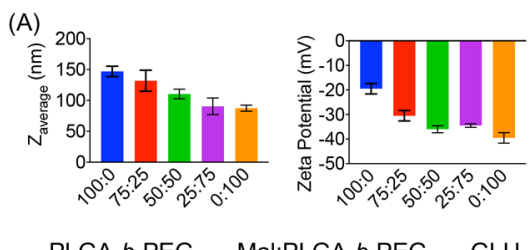

(B)
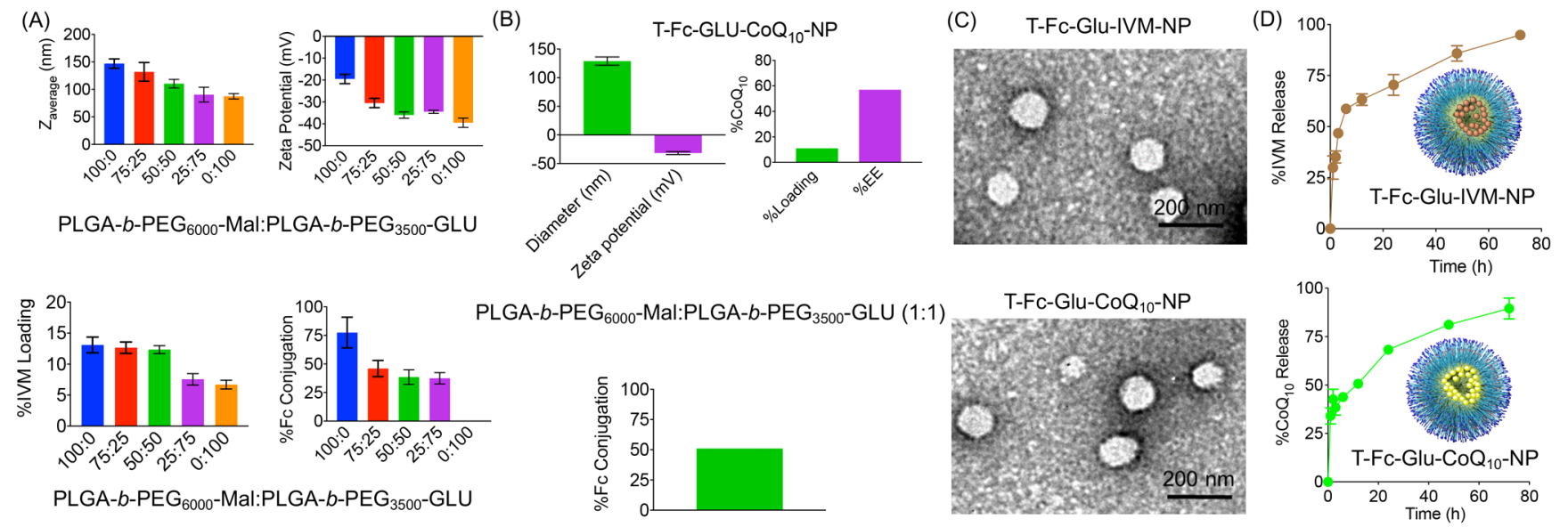

Figure 3. (A) Size, $\zeta$ potential, percent IVM loading, and percent $\mathrm{Fc}_{\mathrm{c}}$ conjugation of NP library made with varying PLGA- $b$-PEG $6000-\mathrm{Mal}$ to PLGA$b$ - $\mathrm{PEG}_{3500}$-GLU ratios with $20 \%$ feed of IVM to optimize the properties. (B) Size, $\zeta$ potential, percent loading, EE, and percent $\mathrm{Fc}$ conjugation of a $\mathrm{CoQ}_{10}$-loaded dual-targeted nanoparticle made with 1:1 PLGA- $b-\mathrm{PEG}_{6000}-\mathrm{Mal}$ to PLGA- $b$-PEG 3500 -GLU and 20\% feed of CoQ ${ }_{10}$. (C) Analyses of morphology and diameter of dual targeted NPs using TEM. (D) Release kinetics of IVM (top) and CoQ ${ }_{10}$ (bottom) from their respective dual targeted NPs under physiological conditions of $\mathrm{pH} 7.4$ at $37^{\circ} \mathrm{C}$.

PLGA- $b-$ PEG $_{3500}$-GLU, using an urea based ligand GLU to result targeted NPs to adequately target these tissues in AKIlike conditions once in the bloodstream. Thus, in this study, the plan was to synthesize a blended NP made up of two polymers with unique targeting capabilities. This first requires the synthesis of PLGA- $b-\mathrm{PEG}_{6000}-\mathrm{Mal}$, such that the longer PEG chain with molecular weight 6000 will allow for the conjugated Fc fragments to exist more prominently on the NP's surface. This is crucial, as the Fc fragment comprises the first of two targeting mechanisms in the dual-targeted NP and binding of gut FcRn receptors must be prioritized before the kidney-targeting mechanism can be utilized. The second polymer, PLGA- $b$-PEG 3500 -GLU with a shorter PEG chain length was conjugated to GLU ligand that will bind to PSMA receptors in kidney cells, so that the NP can be taken up and deliver IVM and $\mathrm{CoQ}_{10}$ to SARS-CoV-2-infected kidney cells. The composition of the NP was optimized to develop an appropriate balance between the two polymers, in order to maintain an ideal size, $\zeta$ potential, and loading of IVM. To achieve dual-targeting ability, two polymers were synthesized to incorporate unique active targeting mechanisms and a series of NPs were constructed including NPs made with PLGA- $b$ $\mathrm{PEG}_{6000}$-Mal only to result T-Fc-IVM-NP, PLGA- $b-\mathrm{PEG}_{3500^{-}}$ GLU only to make T-GLU-IVM-NP, and both polymers to create T-Fc-GLU-IVM-NP (Figure 2A). Mal-NHS and PLGA$b$-PEG $6000-\mathrm{NH}_{2}$ were produced and used to synthesize PLGA$b$-PEG $6000-\mathrm{Mal}$, a polymer to which the $\mathrm{Fc}$ immunoglobulin fragment would be attached for targeting of the FcRnexpressing gut epithelial cells (Figure 2B). The second polymer, PLGA- $b$-PEG $3500-G L U,{ }^{26}$ was synthesized using PLGA- $b-\mathrm{PEG}_{3500}-\mathrm{COOH}$ and a modified urea based small ligand, GLU known to bind to the cell surface receptor PSMA. Using these polymers, IVM-loaded NPs were synthesized via nanoprecipitation using $20 \%$ feed of IVM (with respect to total polymer amount) as well PLGA- $b$-PEG 6000 -Mal and PLGA- $b$ $\mathrm{PEG}_{3500}$-GLU (Figure 2C). This was followed by attachment of the Fc antibody fragment using thiol-ene chemistry, in which 2-iminothiolane was used to link the $\mathrm{Fc}_{\mathrm{c}}$ fragment to the PLGA- $b$-PEG 6000 -Mal polymer at the nanoparticle surface via a disulfide linkage (Figure 2C). To optimize the composition of the two polymers within the dual-targeted NP, IVM-loaded
NPs were synthesized with five varying PLGA- $b-\mathrm{PEG}_{6000}-\mathrm{Mal}$ to PLGA- $b$-PEG 3500 -GLU ratios, ranging from 100:0 to 0:100, each with $20 \%$ feed of IVM (Figure S6). Size, $\zeta$ potential, percent loading, and percent encapsulation efficiency were measured for each unique NP composition. These NPs were conjugated with Fc-fragment using thiolene chemistry. Dynamic light scattering (DLS) was used to measure hydrodynamic diameter and $\zeta$ potential; the NPs were found to have decreasing size from 140 to $75 \mathrm{~nm}$ as the amount of the PLGA- $b$-PEG-GLU increased relative to the amount of the PLGA- $b$-PEG-MAL polymer (Figure $3 A$ ). This decrease in size was predicted as it is known that PEG length can influence the diameters of NPs. The $\zeta$ potential was also found to become more negative, from around $-20 \mathrm{mV}$ to $-40 \mathrm{mV}$, as the relative amount of PLGA- $b$-PEG-GLU increased. Percent loading and encapsulation efficiency of IVM into the NPs decreased as the relative amount of PLGA- $b$-PEG-GLU increased in accordance with the decrease in the NP size but was found to be adequately high for NPs containing at least $50 \%$ of PLGA- $b$-PEG-Mal. Overall, the NPs consisting of a 1:1 ratio of the two polymers showed promising results for all of the measured metrics. Dual-targeted NP consisting of PLGA- $b$ $\mathrm{PEG}_{6000}-\mathrm{Mal}$ and PLGA- $b$-PEG 3500 -GLU loaded with the antioxidant and anti-inflammatory agent $\mathrm{CoQ}_{10}$ was also produced. These T-Fc-GLU-CoQ 10 -NPs had hydrodynamic diameter of around $125 \mathrm{~nm}$ and $\zeta$ potential of around $-35 \mathrm{mV}$, and showed effective $\mathrm{Fc}$ conjugation and loading of $\mathrm{CoQ}_{10}$ (Figure 3B). Analyses of morphology of dual targeted IVM and $\mathrm{CoQ}_{10}$ NPs using transmission electron microscopy (TEM) revealed spherical, homogeneous, well-dispersed particle populations (Figure 3C). The diameter of the NPs determined by TEM also follow similar trend as observed by DLS technique. We also studied the release kinetics of IVM and $\mathrm{CoQ}_{10}$ from their respect dual targeted NPs, T-Fc-GLU-IVM$\mathrm{NP}$ and T-Fc-GLU-CoQ ${ }_{10}-\mathrm{NP}$ using a dialysis method. Our results documented controlled release of IVM and $\mathrm{CoQ}_{10}$ from their respective NPs for an extended period under physiological conditions of $\mathrm{pH} 7.4$ and temperature $37{ }^{\circ} \mathrm{C}$ (Figure 3D). Around $60 \%$ of IVM or $\mathrm{CoQ}_{10}$ release was observed in 24 $\mathrm{h}$ and the remaining amount was released in a controlled manner until $72 \mathrm{~h}$. 
(A)

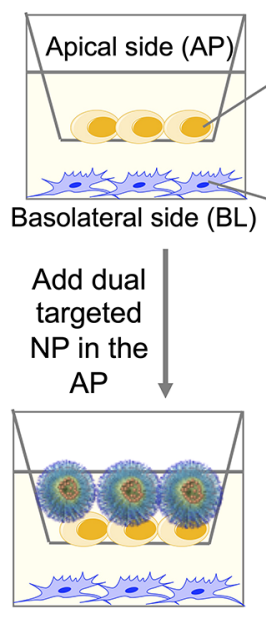

(B)

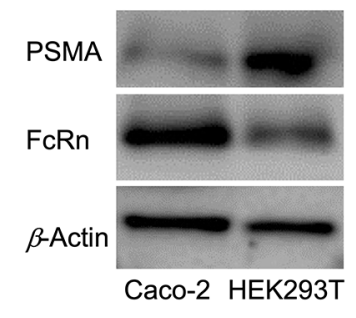

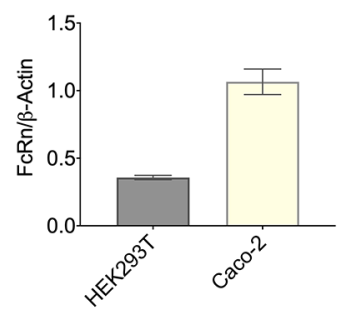

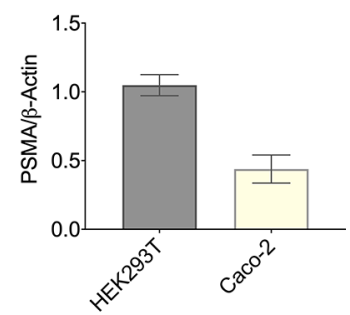

(C)

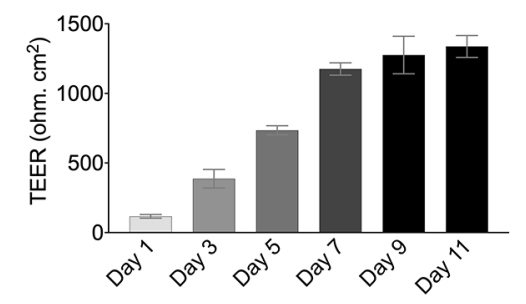

(D)

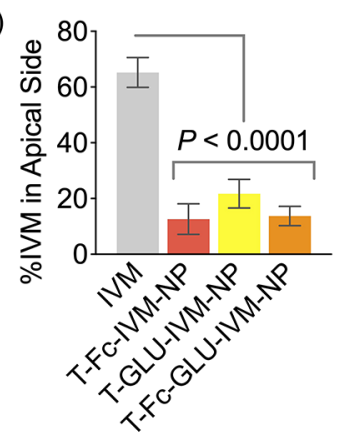

FcRn Expressing epithelial Caco-2 cells

Fc mediated crossing of epithelial barrier
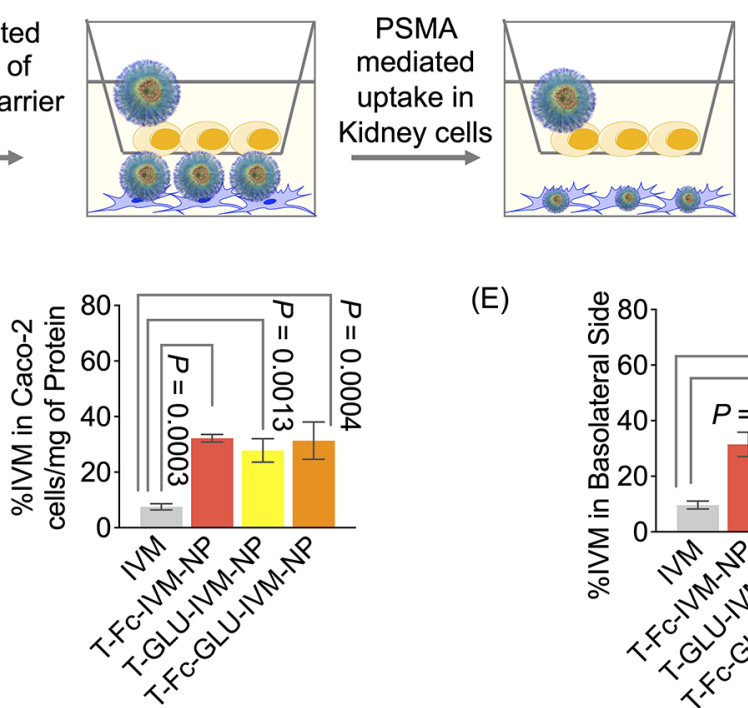

(E)
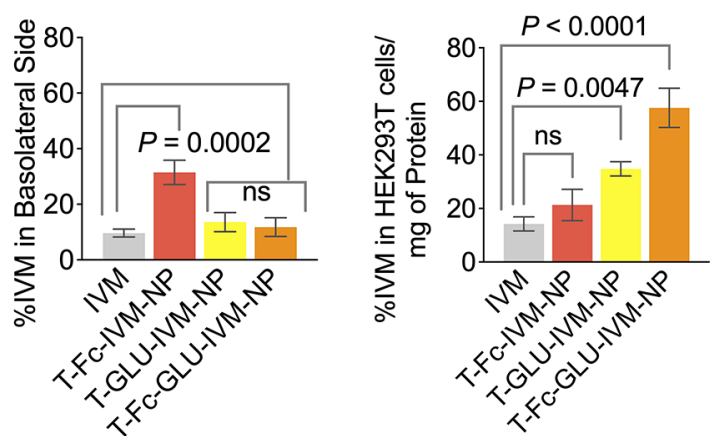

Figure 4. (A) Scheme showing formation of epithelial barrier with Caco-2 cells on apical side and HEK293T cells on basolateral side, followed by Fc-mediated barrier crossing and PSMA-mediated nanoparticle uptake. (B) Western blot showing expression of PSMA and FcRn in HEK293T cells and Caco-2 cells. (C) Barrier formation and integrity as shown by TEER measurements. Figure showing presence of ivermectin in medium and inside cells in (D) apical and (E) basolateral sides.

(A)

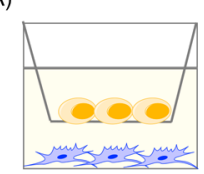

(B)

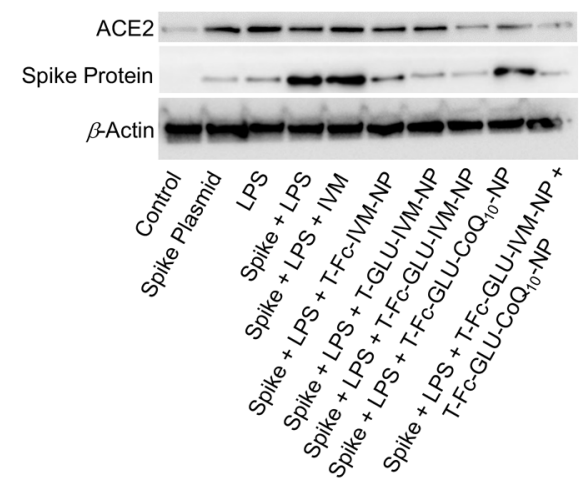

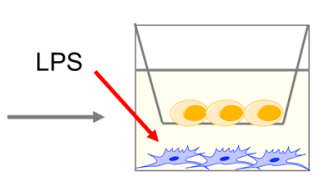

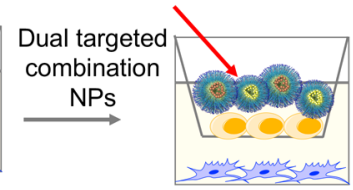

(C)

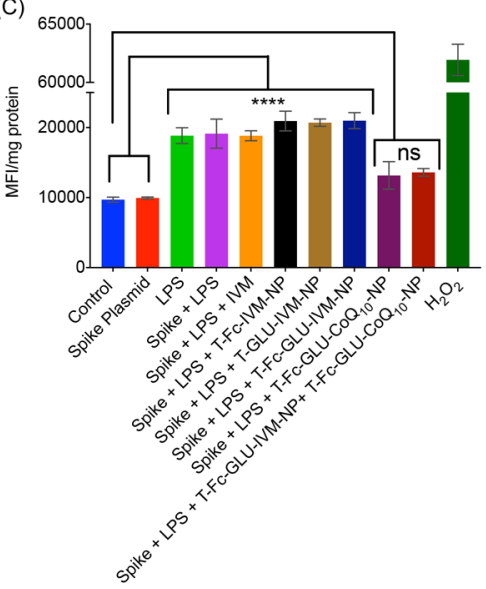

Figure 5. (A) Schematic representation showing the development of a barrier model with Caco-2 cells on the apical side and HEK293T cells on the basolateral side, followed by addition of spike plasmid and/or LPS to HEK293T cells, and treatment with IVM or CoQ 10 -loaded dual-targeted NPs. LPS was added at a concentration of $100 \mathrm{ng} / \mathrm{mL}$. Articles were added to the apical media at a concentration of $10 \mu \mathrm{M}$ with respect to IVM or $\mathrm{CoQ}_{0}$. (B) Western blot showing the changes in expression of ACE2 and spike protein. (C) DCFDA assay showing ROS. 
(A)

NP Administration via oral gavage

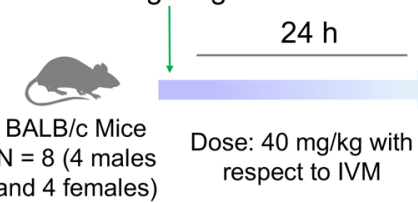

(C) per group

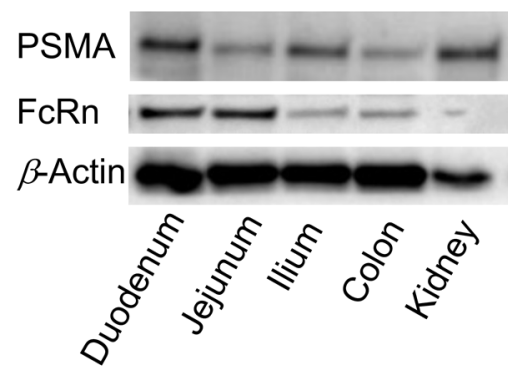

(B) $\mathrm{F}$

Fc75:GLU25 $=$ T-F $\mathrm{C}_{75}$-GLU ${ }_{25}$-IVM-NP

Fc50:GLU50 = T-Fc $\mathrm{C}_{50}-\mathrm{GLU}_{50}$-IVM-NP

Fc25:GLU75 = T-Fc ${ }_{75}-G_{2} U_{25}$ IVM-NP

GLU100 = T-GLU-IVM-NP

Sacrifice to

collect organs

(D)

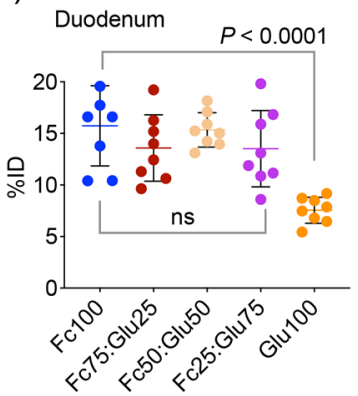

(E)
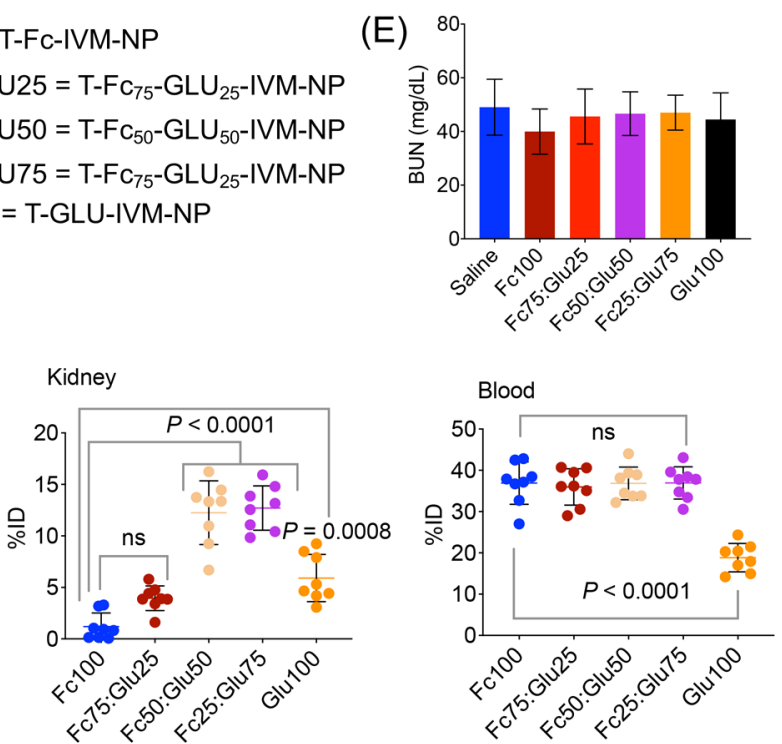

Figure 6. (A) Specifications for biodistribution studies for dual-targeted IVM-loaded NPs of different polymer compositions run with $n=8$ BALB/ c mice in each group and dose of $40 \mathrm{mg} / \mathrm{kg}$ with respect to IVM. (B) NP compositions varying in Fc:GLU polymer ratio, from $100 \%$ Fc polymer (T-Fc-IVM-NP) to $100 \%$ GLU polymer (T-GLU-IVM-NP). (C) Western blot showing expression levels of PSMA and FcRn in duodenum, jejunum, ilium, colon, and kidney of mice. (D) Graphs showing accumulation of the IVM-loaded NPs with varying compositions in duodenum, kidney, and blood of mice. (E) BUN levels of mice treated with IVM-loaded NPs with varying compositions.

In Vitro Evaluation of Blended Nanoparticle's Ability to Cross Multiple Barriers. To test the gut barrier targeting and uptake of the dual-targeted IVM-loaded NPs, a barrier model was developed in transwell plates to model the crossing of gut barrier and targeting of kidney cells (Figure 4A). Caco-2 human colorectal adenocarcinoma cells were seeded on the apical side to mimic the gut epithelial barrier, as these cells express the FcRn receptor needed for the transcytosis of the Fc-conjugated NPs. HEK293T human embryonic kidney cells were seeded on the basolateral side in order to represent the kidney, since these cells also express higher than normal levels of surface PSMA, which the PLGA- $b$-PEG-GLU polymer was expected to target. NPs were synthesized using either the PLGA- $b$-PEG-Mal or PLGA- $b$-PEG-GLU polymers or both polymers in a $1: 1$ ratio, after which sulfhydryl immobilization was used to conjugate $\mathrm{Fc}$ fragments onto the NPs as described before. Western blot analyses confirmed the presence of PSMA on HEK293T cells and FcRn on Caco-2 cells (Figure 4B). Trans-epithelial electrical resistance (TEER) values were collected across multiple days to ensure proper barrier formation and maintenance of barrier integrity (Figure 4C). Quantification of IVM using HPLC showed that while IVM alone was unable to effectively pass through the Caco-2 epithelial barrier to the HEK293T cells on the basolateral side, the dual-targeted T-Fc-GLU-IVM-NPs showed the highest uptake into the kidney cells (Figures 4D and 4E).

Nanoparticle-Mediated Reduction of SARS-CoV-2 Spike Protein and Oxidative Stress in Kidney Barrier Model. To understand the effect of dual-targeted blended NPs containing IVM and $\mathrm{CoQ}_{10}$ on inflammation and reactive oxygen species (ROS) in cells expressing SARS-CoV-2 spike protein, a transwell barrier model consisting of epithelial and kidney cells was developed (Figure 5A). Both the IVM-loaded and $\mathrm{CoQ}_{10}$-loaded NPs were tested in this model to mimic combination treatment meant to reduce COVID-19 uptake and viral transmission as well as inflammation and oxidative stress due to AKI and COVID-19. Furthermore, cells were treated with LPS or transfected with the spike protein plasmid, or received both exposures, to induce COVID-19-related and nonspecific inflammation and ROS. Western blot from basolateral layer HEK293T cells data after exposures and treatment with the therapeutic NPs showed that the dualtargeted IVM-loaded NP was able to reduce both ACE2 and spike protein expression, since it possessed the Fc and GLU to cross the gut barrier and be taken up into the kidney cells, as well as the IVM necessary to reduce levels of ACE2 and spike protein (Figure 5B).

To understand the effects of combination of dual-targeted IVM- and dual-targeted $\mathrm{CoQ}_{10}-\mathrm{NPs}$ on ROS on cells expressing SARS-CoV-2 spike protein, ROS levels were measured via $2^{\prime}, 7^{\prime}$-dichlorofluorescin diacetate (DCFDA) from HEK293T cells from the basolateral side of the aforementioned transwell barrier model (Figure 5C). The presence of LPS and spike protein significantly increased ROS levels above control levels, an effect that was not able to be reversed by treatment with free IVM or targeted IVM nanoformulations alone. When cells were treated either with the $\mathrm{CoQ}_{0}$-loaded T-Fc-GLU-CoQ ${ }_{10}$-NPs alone or in combination with the IVM-loaded T-Fc-GLU-IVM-NPs, ROS levels were reduced comparable to control levels (Figure 5C).

Bioavailability of Blended Dual-targeted IVM-loaded Nanoparticles in Kidney. A biodistribution study was conducted with $\mathrm{BALB} / \mathrm{c}$ mice to optimize the various polymer compositions of the dual-targeted IVM-loaded NPs and their accumulation in various organs and blood in order to understand the potential of the nanoformulation for future use as a therapeutic (Figure 6A). T-Fc-GLU-IVM-NPs made from PLGA-b-PEG $6000-\mathrm{Mal}$ polymer with conjugated $\mathrm{Fc}$ fragment and the PLGA- $b-\mathrm{PEG}_{3500}-\mathrm{GLU}$ polymer with varied compositions from $100 \%$ of either to a mixture of the two in order to test which of the five NPs had optimal biodistribution were used (Figure 6B). To measure the level of receptors in organs that would be targeted by the dual-targeted NPs, PSMA and FcRn levels were analyzed in the gut and kidney of the 
mice using Western blot (Figure 6C). This revealed that levels of $\mathrm{FcRn}$ were higher in earlier portions of the gut, indicating the $\mathrm{Fc}$ fragments conjugated to the NPs would be able to allow for adequate NP transcytosis into the bloodstream after oral administration. In addition, PSMA levels were higher in the kidney of mice, suggesting that the NPs' second targeting mechanism, the GLU portion attached to the PLGA- $b$ $\mathrm{PEG}_{3500}-\mathrm{GLU}$ polymer, would allow for appropriate uptake into the kidney cells for delivery of IVM. Analysis of the mice organs revealed that all NPs with Fc conjugation were able to reach the duodenum and accumulate in the bloodstream at levels of around $40 \%$, meaning they would be in circulation and able to reach organs and sites of infection and injury (Figure 6D). However, only the NPs with a 50:50 or 25:75 ratio of PLGA- $b$-PEG 6000 -Mal to PLGA- $b-$ PEG $_{3500}$-GLU were able to adequately accumulate in the kidney of the treated mice, indicating that the dual-targeted IVM-loaded NPs need to consist of at least 50\% PLGA- $b$-PEG 3500 -GLU to fully exploit the PSMA targeting mechanism and be taken up into the kidney cells. Analyses of blood urea nitrogen (BUN) levels of mice to test the toxicity of the nanoformulations on the kidney revealed that none of the nanoformulations increased BUN levels past the control level of $45-50 \mathrm{mg} / \mathrm{dL}$ (Figure $6 \mathrm{E})$.

\section{CONCLUSIONS}

The gut barrier-penetrating and kidney-targeting nature of the NP provided by the blending of PLGA- $b-$ PEG $_{6000}-\mathrm{Mal}$ and PLGA- $b$-PEG 3500 -GLU polymers as well as the Fc fragment conjugation will be suitable for a variety of therapeutics meant to treat the SARS-CoV-2 infection as well as the oxidative stress and inflammation associated with the kidney damage. The primary polymer design considers the necessary sequence of targeting mechanisms, as the longer PLGA- $b-\mathrm{PEG}_{6000}-\mathrm{Mal}$ polymer's Fc-mediated gut transcytosis will occur prior to the shorter PLGA- $b$-PEG 3500 -GLU polymer's PSMA targeting once the NP is in the bloodstream. Through optimization of the two-polymer composition of the NP, in vitro barrier penetration studies, in vitro efficacy studies, and in vivo biodistribution and toxicity studies, we were able to successfully synthesize and fine-tune the nanoformulation to effectively accumulate at the kidney: an effect that will be augmented due to any tissue injury or inflammation that may be present at the organ. The nanoformulation was able to achieve several key goals, including penetration through the gut barrier and kidney barrier and reduction in expression of ACE2 and spike protein as well as ROS levels. This nanoplatform is a promising potential drug delivery tool for patients suffering from kidney damage and long-term effects of COVID-19.

\section{ASSOCIATED CONTENT}

\section{SI Supporting Information}

The Supporting Information is available free of charge at https://pubs.acs.org/doi/10.1021/acs.biomac.1c00799.

Detailed description of materials and methods, additional figures (PDF)

\section{AUTHOR INFORMATION}

\section{Corresponding Author}

Shanta Dhar - Department of Biochemistry and Molecular Biology, Leonard M. Miller School of Medicine and Sylvester
Comprehensive Cancer Center, Leonard M. Miller School of Medicine, University of Miami, Miami, Florida 33136, United States; Department of Chemistry, University of Miami, Coral Gables, Florida 33146, United States; ○ orcid.org/0000-0003-3042-5272; Email: shantadhar@ med.miami.edu

\section{Authors}

Bapurao Surnar - Department of Biochemistry and Molecular Biology, Leonard M. Miller School of Medicine and Sylvester Comprehensive Cancer Center, Leonard M. Miller School of Medicine, University of Miami, Miami, Florida 33136, United States

Anuj S. Shah - Department of Biochemistry and Molecular Biology, Leonard M. Miller School of Medicine, University of Miami, Miami, Florida 33136, United States

Subham Guin - Department of Biochemistry and Molecular Biology, Leonard M. Miller School of Medicine, University of Miami, Miami, Florida 33136, United States

Nagesh Kolishetti - Department of Immunology and NanoMedicine, Herbert Wertheim College of Medicine, Florida International University, Miami, Florida 33199, United States; orcid.org/0000-0001-5574-673X

Alessia Fornoni - Katz Family Division of Nephrology and Hypertension, Department of Medicine and Peggy and Harold Katz Family Drug Discovery Center, Department of Medicine, University of Miami Miller School of Medicine, Miami, Florida 33136, United States

Complete contact information is available at:

https://pubs.acs.org/10.1021/acs.biomac.1c00799

\section{Author Contributions}

S.D. conceptualized, supervised the research, and provided directions and resources; S.D, B.S., A.S. and N.K. designed experiments; B.S., A.S., and S.G. performed research; B.S., and A.S. contributed reagents; S.D., B.S., A.S., N.K. and A.F. analyzed the data. All authors discussed the results and commented on the manuscript; B.S., A.S., N.K., A.F., and SD wrote the manuscript.

\section{Notes}

The authors declare the following competing financial interest(s): A.F. is Vice President of L\&F Health LLC and is a consultant for ZyVersa Therapeutics, Inc. Other authors declare no conflict of interests with the technologies mentioned here.

\section{ACKNOWLEDGMENTS}

This work was supported by Sylvester Comprehensive Cancer Center, University of Miami Miller School of Medicine to S.D. We also thank the National Institutes of Health (NIH) grant DA044579 and R01CA227493. A.F. acknowledges NIH grants R01DK117599, R01DK104753, and R01CA227493.

\section{REFERENCES}

(1) Ou, X.; Liu, Y.; Lei, X.; Li, P.; Mi, D.; Ren, L.; Guo, L.; Guo, R.; Chen, T.; Hu, J.; Xiang, Z.; Mu, Z.; Chen, X.; Chen, J.; Hu, K.; Jin, Q.; Wang, J.; Qian, Z. Characterization of spike glycoprotein of SARS$\mathrm{CoV}-2$ on virus entry and its immune cross-reactivity with SARS-CoV. Nat. Commun. 2020, 11, 1620.

(2) Sungnak, W.; Huang, N.; Bécavin, C.; Berg, M.; Queen, R.; Litvinukova, M.; Talavera-López, C.; Maatz, H.; Reichart, D.; Sampaziotis, F.; Worlock, K. B.; Yoshida, M.; Barnes, J. L.; Network, H. L. B. SARS-CoV-2 entry factors are highly expressed 
in nasal epithelial cells together with innate immune genes. Nat. Med. 2020, 26, 681-687.

(3) Nugent, J.; Aklilu, A.; Yamamoto, Y.; Simonov, M.; Li, F.; Biswas, A.; Ghazi, L.; Greenberg, J. H.; Mansour, S. G.; Moledina, D. G.; Wilson, F. P. Assessment of Acute Kidney Injury and Longitudinal Kidney Function After Hospital Discharge Among Patients With and Without COVID-19. JAMA Netw. Open. 2021, 4 (3), e211095.

(4) Nadim, M. K.; Forni, L. G.; Mehta, R. L.; Connor, M. J., Jr.; Liu, K. D.; Ostermann, M.; Rimmele, T.; Zarbock, A.; Bell, S.; Bihorac, A.; Cantaluppi, V.; Hoste, E.; Husain-Syed, F.; Germain, M. J.; Goldstein, S. L.; Gupta, S.; Joannidis, M.; Kashani, K.; Koyner, J. L.; Legrand, M.; Lumlertgul, N.; Mohan, S.; Pannu, N.; Peng, Z.; Perez-Fernandez, X. L.; Pickkers, P.; Prowle, J.; Reis, T.; Srisawat, N.; Tolwani, A.; Vijayan, A.; Villa, G.; Yang, L.; Ronco, C.; Kellum, J. A. COVID-19associated acute kidney injury: consensus report of the 25th Acute Disease Quality Initiative (ADQI) Workgroup. Nat. Rev. Nephrol. 2020, 16 (12), 747-764.

(5) Shao, M.; Li, X.; Liu, F.; Tian, T.; Luo, J.; Yang, Y. Acute kidney injury is associated with severe infection and fatality in patients with COVID-19: A systematic review and meta-analysis of 40 studies and 24,527 patients. Pharmacol. Res. 2020, 161, 105107.

(6) Bucaloiu, I. D.; Kirchner, H. L.; Norfolk, E. R.; Hartle, J. E., 2nd; Perkins, R. M. Increased risk of death and de novo chronic kidney disease following reversible acute kidney injury. Kidney Int. 2012, 81 (5), 477-85.

(7) Cola, D. F.; Pasquoto, T.; Guilger, M.; Lima, R. d.; Silva, C. M. G. d.; Fraceto, L. F. Lipid Nanostructured Carriers Systems for Ivermectin and Methoprene Aiming Parasite Control. Quim. Nova. 2016, 39, 1034-1043.

(8) Joseph, A.; Zafrani, L.; Mabrouki, A.; Azoulay, E.; Darmon, M. Acute kidney injury in patients with SARS-CoV-2 infection. Ann. Intensive Care 2020, 10 (1), 117.

(9) Kudose, S.; Batal, I.; Santoriello, D.; Xu, K.; Barasch, J.; Peleg, Y.; Canetta, P.; Ratner, L. E.; Marasa, M.; Gharavi, A. G.; Stokes, M. B.; Markowitz, G. S.; D’Agati, V. D. Kidney Biopsy Findings in Patients with COVID-19. J. Am. Soc. Nephrol. 2020, 31 (9), 1959-1968.

(10) Makris, K.; Spanou, L. Acute Kidney Injury: Definition, Pathophysiology and Clinical Phenotypes. Clin Biochem Rev. 2016, 37 (2), 85-98.

(11) Chalikias, G.; Serif, L.; Kikas, P.; Thomaidis, A.; Stakos, D.; Makrygiannis, D.; Chatzikyriakou, S.; Papoulidis, N.; Voudris, V.; Lantzouraki, A.; Muller, M.; Arampatzis, S.; Konstantinides, S.; Tziakas, D. Long-term impact of acute kidney injury on prognosis in patients with acute myocardial infarction. Int. J. Cardiol. 2019, 283, $48-54$.

(12) Gadalean, F.; Simu, M.; Parv, F.; Vorovenci, R.; Tudor, R.; Schiller, A.; Timar, R.; Petrica, L.; Velciov, S.; Gluhovschi, C.; Bob, F.; Mihaescu, A.; Timar, B.; Spasovski, G.; Ivan, V. The impact of acute kidney injury on in-hospital mortality in acute ischemic stroke patients undergoing intravenous thrombolysis. PLoS One 2017, 12 (10), No. e0185589.

(13) Nuthalapati, A.; Schluterman, N.; Khanna, A.; Greenberg, D.; Thuluvath, P. J. Impact of Acute Kidney Injury on Mortality of Patients Hospitalized for Complications of Cirrhosis. J. Clin. Exp. Hepatol. 2017, 7 (4), 290-299.

(14) Rodriguez, E.; Arias-Cabrales, C.; Bermejo, S.; Sierra, A.; Burballa, C.; Soler, M. J.; Barrios, C.; Pascual, J. Impact of Recurrent Acute Kidney Injury on Patient Outcomes. Kidney Blood Pressure Res. 2018, 43 (1), 34-44.

(15) Peng, S.; Wang, H. Y.; Sun, X.; Li, P.; Ye, Z.; Li, Q.; Wang, J.; Shi, X.; Liu, L.; Yao, Y.; Zeng, R.; He, F.; Li, J.; Ge, S.; Ke, X.; Zhou, Z.; Dong, E.; Wang, H.; Xu, G.; Zhang, L.; Zhao, M. H. Early versus late acute kidney injury among patients with COVID-19-a multicenter study from Wuhan, China. Nephrol., Dial., Transplant. 2020, 35 (12), 2095-2102.

(16) Surnar, B.; Kamran, M. Z.; Shah, A. S.; Basu, U.; Kolishetti, N.; Deo, S.; Jayaweera, D. T.; Daunert, S.; Dhar, S. Orally Administrable Therapeutic Synthetic Nanoparticle for Zika Virus. ACS Nano 2019, 13, 11034-11048.
(17) Surnar, B.; Kamran, M. Z.; Shah, A. S.; Dhar, S. Clinically Approved Antiviral Drug in an Orally Administrable Nanoparticle for COVID-19. ACS Pharmacol Transl Sci. 2020, 3 (6), 1371-1380.

(18) Dickinson, B. L.; Badizadegan, K.; Wu, Z.; Ahouse, J. C.; Zhu, X.; Simister, N. E.; Blumberg, R. S.; Lencer, W. I. Bidirectional FcRndependent IgG transport in a polarized human intestinal epithelial cell line. J. Clin. Invest. 1999, 104 (7), 903-11.

(19) Yoshida, M.; Claypool, S. M.; Wagner, J. S.; Mizoguchi, E.; Mizoguchi, A.; Roopenian, D. C.; Lencer, W. I.; Blumberg, R. S. Human neonatal $\mathrm{Fc}$ receptor mediates transport of IgG into luminal secretions for delivery of antigens to mucosal dendritic cells. Immunity 2004, 20 (6), 769-83.

(20) Israel, E. J.; Taylor, S.; Wu, Z.; Mizoguchi, E.; Blumberg, R. S.; Bhan, A.; Simister, N. E. Expression of the neonatal Fc receptor, FcRn, on human intestinal epithelial cells. Immunology 1997, 92 (1), 69-74.

(21) Al-Ahmadie, H. A.; Olgac, S.; Gregor, P. D.; Tickoo, S. K.; Fine, S. W.; Kondagunta, G. V.; Scher, H. I.; Morris, M. J.; Russo, P.; Motzer, R. J.; Reuter, V. E. Expression of prostate-specific membrane antigen in renal cortical tumors. Mod. Pathol. 2008, 21 (6), 727-732.

(22) Baccala, A.; Sercia, L.; Li, J.; Heston, W.; Zhou, M. Expression of prostate-specific membrane antigen in tumor-associated neovasculature of renal neoplasms. Urology 2007, 70 (2), 385-90.

(23) Morgantetti, G.; Ng, K. L.; Samaratunga, H.; Rhee, H.; Gobe, G. C.; Wood, S. T. Prostate specific membrane antigen (PSMA) expression in vena cava tumour thrombi of clear cell renal cell carcinoma suggests a role for PSMA-driven tumour neoangiogenesis. Transl. Androl. Urol. 2019, 8 (S2), S147-S155.

(24) Rhee, H.; Ng, K. L.; Tse, B. W.; Yeh, M. C.; Russell, P. J.; Nelson, C.; Thomas, P.; Samaratunga, H.; Vela, I.; Gobe, G.; Wood, $\mathrm{S}$. Using prostate specific membrane antigen (PSMA) expression in clear cell renal cell carcinoma for imaging advanced disease. Pathology 2016, 48 (6), 613-6.

(25) Silver, D. A.; Pellicer, I.; Fair, W. R.; Heston, W. D.; CordonCardo, C. Prostate-specific membrane antigen expression in normal and malignant human tissues. Clin. Cancer Res. 1997, 3 (1), 81-5.

(26) Pathak, R. K.; Basu, U.; Ahmad, A.; Sarkar, S.; Kumar, A.; Surnar, B.; Ansari, S.; Wilczek, K.; Ivan, M. E.; Marples, B.; Kolishetti, N.; Dhar, S. A designer bow-tie combination therapeutic platform: An approach to resistant cancer treatment by simultaneous delivery of cytotoxic and anti-inflammatory agents and radiation. Biomaterials 2018, 187, 117-129. 